SCIENTIFIC AMERICANS 



\section{SCIENTIFIC AMERICANS}

\section{INVENTION, TECHNOLOGY, AND NATIONAL IDENTITY}

\section{SUSAN BRANSON}


Copyright (C) 2021 by Cornell University

All rights reserved. Except for brief quotations in a review, this book, or parts thereof, must not be reproduced in any form without permission in writing from the publisher. For information, address Cornell University Press, Sage House, 512 East State Street, Ithaca, New York 14850. Visit our website at cornellpress.cornell.edu.

First published 2021 by Cornell University Press

Printed in the United States of America

Library of Congress Cataloging-in-Publication Data

Names: Branson, Susan, author.

Title: Scientific Americans : invention, technology, and national identity / Susan Branson.

Description: Ithaca [New York] : Cornell University Press, 2021. Includes bibliographical references and index.

Identifiers: LCCN 2021032218 (print) | LCCN 2021032219 (ebook) | ISBN 9781501760914 (hardcover) | ISBN 9781501760921 (pdf) | ISBN 9781501760938 (epub)

Subjects: LCSH: Science-Social aspects—United States-History-19th century. | Technology—Social aspects—United States—History-19th century. | Science-Social aspects-United States-History18th century. $\mid$ Technology-Social aspects-United States-History-18th century.

Classification: LCC Q175.52.U5 B73 2022 (print) | LCC Q175.52.U5 (ebook) | DDC 303.48/30973—dc23

LC record available at https://lccn.loc.gov/ 2021032218

LC ebook record available at https: / / lccn.loc.gov/2021032219

Cover image details (colorized): Clockwise from upper-left corner: "Balloon ascension to the Presidential Chair" (1844, Library of Congress); "Mr. Golightly, Bound to California" (ca. 1849, Library of Congress); "The Way They Go to California" (ca. 1849, Library of Congress); "Locomotion: Walking by Steam" (ca. 1830, Lewis Walpole Library, Yale University); "Locomotion: Walking by Steam"; "Locomotion: Walking by Steam." See interior figure captions for full credit information. 
For Mark 
